\title{
Performance evaluation of a high-resolution parallel-plate differential mobility analyzer
}

\author{
J. P. Santos ${ }^{1}$, E. Hontañón ${ }^{1}$, E. Ramiro ${ }^{1}$, and M. Alonso ${ }^{2}$ \\ ${ }^{1}$ RAMEM S.A., Sambara 33, 28027 Madrid, Spain \\ ${ }^{2}$ National Center for Metallurgical Research (CSIC), Avda. Gregorio del Amo 8, 28040 Madrid, Spain \\ Received: 17 June 2008 - Published in Atmos. Chem. Phys. Discuss.: 29 September 2008 \\ Revised: 4 February 2009 - Accepted: 26 March 2009 - Published: 3 April 2009
}

\begin{abstract}
A high-resolution differential mobility analyzer (DMA), specially designed for (i) the measurement of ion mobility spectra, and (ii) the generation of a continuous stream of monomobile ions, has been developed and tested. The apparatus consists of two parallel-plate electrodes between which an electric field is applied. The test ion stream flows into the instrument through a narrow rectangular slit made in one of the electrodes, and migrates toward the other electrode driven by the applied field, while being transported by a stream of clean air which flows parallel to the plates at Reynolds number between $2 \times 10^{4}$ and $9 \times 10^{4}$ in laminar flow conditions. The collector electrode contains also a narrow slit through which ions of the desired mobility are withdrawn out of DMA. The classified ion current is measured with a highsensitivity electrometer having a noise level around $0.1 \mathrm{fA}$.

The theory behind the DMA operation is first discussed, focusing on the special case of parallel-plate geometry. Some generic results showing the stability and repeatability of the measurements and the resolving power of the instrument are presented next. The last part of the paper deals with the application of the apparatus to the study of the effect of humidity and aging time on the mobility spectra of air ions generated by a low-activity ${ }^{241} \mathrm{Am}$ source.
\end{abstract}

\section{Introduction}

The mobility of a cluster ion can provide information about its structure and geometry; for instance, geometric isomers

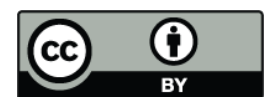

Correspondence to: E. Hontañón (e.hontanon@ramem.com) can be resolved on the basis of their different mobilities (Zarpas et al., 1986, 1988). Ion mobility spectrometry has been used to detect drugs, explosives, chemical warfare agents and other ambient pollutants (Chen et al., 1996; Swing et al., 2001; Hill and Martin, 2002; Buryakov, 2004; Vautz et al., 2006). Traditionally, ion mobility measurements have been performed in drift tubes, containing a buffer gas (maintained at either very low or atmospheric pressure) and a series of electrodes to provide a uniform electric field (Clemmer and Jarrold, 1997). In these instruments, mobility is inferred from the measure time required for an ion to traverse the length of the drift tube.

An alternative instrument to measure ion mobility at atmospheric pressure is the so called differential mobility analyzer (DMA), in which the ions migrate between two electrodes held at different potentials while been transported by a stream of gas (initially clean) flowing parallel to the electrodes. The long history of differential mobility analyzers can be traced back to the early works of Zeleny (1898) and Langevin $(1902,1903)$ using coaxial cylindrical condensers to measure the mobility of air ions. These works, as well as later developments that followed, have been extensively described in a review article of Flagan (1998). In all these early works, the DMA resolution was relatively poor, so that ions with similar mobilities could not be properly resolved.

DMAs later evolved into somewhat longer coaxial cylindrical columns able to measure the mobility of larger aerosol particles and, at the same time, to classify aerosols according to their size. There are two main types of coaxial DMAs, which can be termed single-channel and multi-channel analyzers. In the single-channel DMA, the sampled aerosol exits the apparatus through an outlet slit; the number concentration of the classified particles can then be measured using either

Published by Copernicus Publications on behalf of the European Geosciences Union. 
an electrometer or a particle counter. By varying the electric field between the electrodes, particles of different mobilities are sequentially classified. In this manner, the mobility distribution of the aerosol population can be measured, and can later be transformed into the corresponding particle size distribution provided the charge distribution on the particles is known. The typical prototype of single-channel, coaxial cylindrical DMA was developed in the 1960's at the University of Minnesota (Whitby and Clark, 1966; Knutson and Whitby, 1975).

In the multi-channel design, one of the electrodes is equipped with a series of insulated metal rings, each connected to its own electrometer. When a certain electric field is applied between the coaxial electrodes, the charged particles of a given polarity are deposited differentially onto the metal rings. This arrangement allows for the simultaneous measurement of the number concentration of particles having different mobility, thus reducing considerably the time required for the measurement of the entire mobility distribution. The multi-channel analyzer, which is specially indicated for rapidly fluctuating aerosols, was developed in the 1970's at the University of Tartu (Tammet, 1970; Tammet et al., 2002).

Perhaps the main advantage of the single-channel DMA over multi-channel analyzers and drift tubes is, besides its lower cost, the possibility to use it to generate a continuous stream of monodisperse aerosol particles or monomobile ions. The particles or ions of the desired mobility can subsequently be employed to perform a great variety of fundamental and applied investigations (ion clustering, chemical kinetics, particle size growth by condensation or coagulation, size-dependent electrical behavior, filtration, and many others). (In the rest of this article, we will always have in mind single-channel analyzers, and will refer to them simply as DMAs, omitting the "single-channel" term.)

During the last fifteen years, a great effort has been addressed to the improvement of the performance of DMAs, mainly modifying the aerosol inlet geometry in order to reduce diffusional deposition losses (Winklmayr et al., 1991; Chen et al., 1999), and shortening the mean aerosol residence time by minimizing the column length (Rosell-Llompart et al., 1996) and operating the DMA in laminar flow conditions with sheath gas flow at high Reynolds for classifying ions with high resolution (de Juan and Fernández de la Mora, 1998). Also, two new conceptions of analyzers have appeared, one in which an additional electric field parallel to the main gas flow is applied (Loscertales, 1998), and other type of DMA incorporating an electrified screen which permits maintaining the electrodes containing the inlet and outlet slits at the same potential (Martínez-Lozano et al., 2005, 2006), thus preventing electrophoretic losses.

All the above mentioned improvements were implemented in concentric cylindrical DMAs. With this geometry, electrode centering is critical to the performance of a DMA; even a very small misalignment of the electrodes can lead to a se- vere deterioration of the resolving power of the instrument. To avoid this difficulty, we have preferred to redesign the DMA using parallel-plate geometry, so that electrode centering is no longer an issue. An additional reason for choosing the parallel-plate geometry is related to the strong Brownian motion of nanoparticles and ions. In general, the Brownian motion makes the particles or ions to deviate from their otherwise deterministic trajectories given by the electric and gas flow velocity fields. As a consequence, the trajectories of particles of different mobilities get mixed up and the instrument resolution deteriorates. The extent of Brownian dispersion depends on the uniformity of the electric field and on the average direction of particle motion in the field (Alonso, 2002; Song and Dhaniyala, 2007). Thus, if particles move in the direction of increasing electric field strength, which is the usual case in cylindrical DMAs, the resolution worsens because the variance of the distribution of particle positions is larger than for a free particle, i.e. larger than $2 D t$ ( $D$ is the particle or ion diffusion coefficient, and $t$ is its mean residence time between the inlet and outlet slits). If the electric field is uniform, as in the parallel-plate geometry, the variance is exactly $2 D t$. Other issues like the mechanical machining and positioning of the electrodes are also important. They are both easier and more precise for DMAs of planar plates than for cylindrical DMAs, thereby reducing the cost of the instrument and enhancing its acceptance by potential users.

The first parallel-plate differential mobility classifier was produced by Erikson in 1918. This instrument was used to measure the mobility of gas ions produced by a radioactive source (Erikson, 1921) and the size of smoke and fog particles (Rohmann, 1923). More recently, Zhang and Wexler (2006) described a modern, miniaturized version of a multichannel parallel-plate DMA suitable for analyzing gas phase compounds or volatile particle phase compounds. In these DMAs the electrodes were rectangular planar plates. Circular disks are used as electrodes in radial DMAs like the ones used for the sizing of ultrafine particles (RDMA, Zhang et al., 1995, Zhang and Flagan, 1996; SMEC, Fissan et al., 1996) or for classifying low mobility nanoparticles and ions (Nano-RDMA, Brunelli et al., 2009).

In this work, the novel high-resolution DMA of rectangular planar electrodes developed by us is described in some detail. The electro-fluid-dynamical equations behind the DMA operation will be presented for the specific design of our instrument. Next, the repeatability and stability of the mobility distribution measurements and the resolving power of the DMA will be discussed. The final section of the article will deal with an illustrative application of our DMA: the examination of the effect of humidity and aging time on the mobility distribution of laboratory air ions generated by a ${ }^{241} \mathrm{Am}$ radioactive source. 


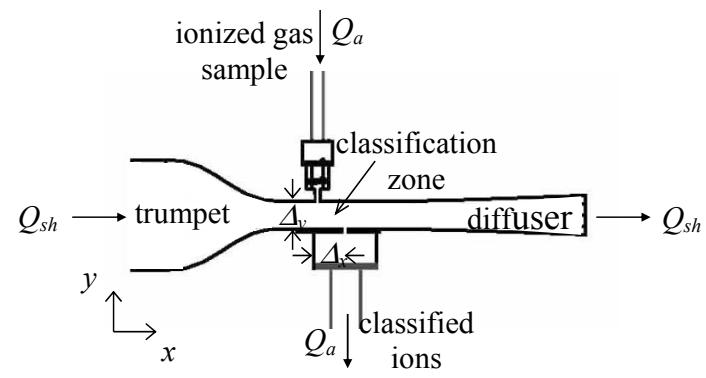

(a)

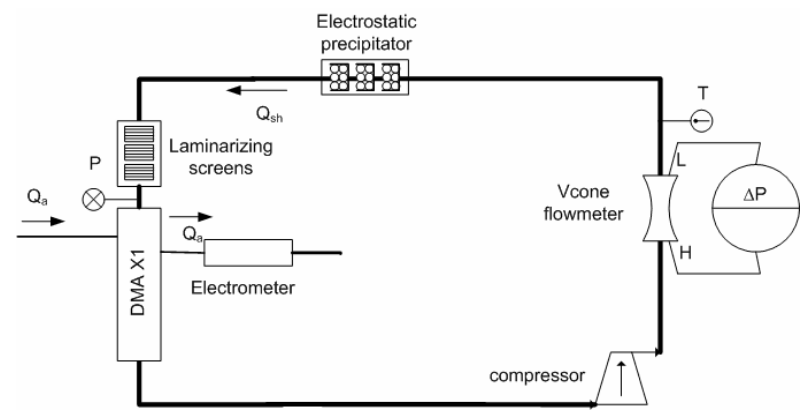

(b)

Fig. 1. Sketch of the IONER X1 Differential Mobility Analyzer: (a) trumpet, classification zone and diffuser; (b) the close-loop circuit of the sheath gas with its accessories.

\section{Description of the instrument}

A sketch of the proposed DMA (RAMEM IONER X1) (Ramiro and Rivero, 2007) is shown in Fig. 1a. The DMA proper, i.e. the classification zone comprising the space delimited by the parallel-plate electrodes and the inlet and outlet slits, is inserted within a closed-circuit rectangular duct in which a sheath gas (usually air) flows. The separation between the electrodes is $5 \mathrm{~mm}$, the distance from the ion inlet slit to the ion outlet slit is also $5 \mathrm{~mm}$ and the width of the classification region is $10 \mathrm{~mm}$. The Reynolds number of the flow in the classification region ranges from $2 \times 10^{4}$ and $9 \times 10^{4}$ (flow rates between 200 and $9001 \mathrm{~min}^{-1}$ ). In spite of these unusually high Reynolds numbers, transition to turbulence could be avoided by means of several stages of laminarizing screens followed by a converging section (trumpet). The screens serve to damp the turbulence of the sheath gas and to spread this uniformly over the flow cross section, while the trumpet helps to stabilize the flow by gradually accelerating it up to the classification zone. The contraction is only in the height dimension and the inlet to outlet area ratio of the trumpet is 8 . Both features cause a noticeable flow pressure drop which is recovered in a diverging section (diffuser) located downstream of the classification region.
The aerosol of ions (or charged nanoparticles) is continuously fed at a low flow rate (generally, less than $61 \mathrm{~min}^{-1}$ ) into the classification zone through a narrow slit. The motion of ions in the classification zone has two components: one parallel to the electrodes, due to the main gas flow; and another one, driven by the applied electric field, directed toward the collector electrode. The latter contains also a slit through which ions having mobility within a narrow range are withdrawn from the loop circuit. The mobility of the thus classified ions depends on the sheath (main) gas flow rate and the strength of the electric field.

If the classified ions are allowed to leave the DMA through the outlet port, the instrument works as a generator of monomobile ions. The possibility of employing this instrument as a stable, continuous source of well-characterized ions is, as commented above, a great advantage of DMAs over time-of-flight mobility spectrometers.

If, on the contrary, one wishes to determine the mobility distribution of the fed ion stream, a metal rod connected to a high-sensitivity electrometer (RAMEM IONER EL-5010) is placed just behind the outlet slit, to measure the current carried by the ions classified at progressively increasing values of the applied electric field.

The dimensions of the inlet and outlet slits can be changed at will. For the experimental work discussed below, we have used rectangular inlet and oulet slits $6 \mathrm{~mm}$ in length and $120 \mu \mathrm{m}$ in width.

At a given value of the applied voltage between the electrodes, only a fraction of ions can at most be classified and withdrawn from the DMA; the rest of the ion population leaves the classification zone with the main gas flow. A fraction of these unclassified, excess ions will progressively be lost by diffusion to the inner walls of the loop circuit, but a certain finite fraction of ions will survive and end up again in the classification zone. A steady buildup of excess ions in the loop would lead to increasing noise in the measured currents. To avoid this undesired effect, the loop duct is equipped with a small electrostatic precipitator downstream of the classification zone, to remove the unclassified ions.

The sheath gas loop circuit is also equipped with a Venturitype flowmeter (McCrometer V-Cone), placed upstream of the classification zone. Pressure and temperature are also measured in the section containing the flowmeter. The actual sheath flow rate in the classification zone is calculated from the values of pressure, temperature and flow rate measured upstream, assuming isentropic flow conditions. It will be shown below that this assumption is fully justified. As the DMA operates in closed-loop circuit the temperature of the sheath gas tends to increase with operating time. A fan was placed above the section between the pump and the flowmeter to cool the gas. This simple setup is enough to keep the gas temperature constant due to the good heat transfer through the metallic walls of the sheath loop. A layout of the ion differential mobility analyzer IONER X1 is depicted in Fig. 1b. 


\subsection{Ideal operation of the parallel-plate DMA}

The operation of a DMA is described by its transfer function $\Omega(k, V)$, which is defined as the probability that an ion of mobility $k$ is classified through the outlet slit when a voltage difference $V$ is applied between the electrodes. To calculate the transfer function of the instrument there is a standard method, first proposed by Knutson and Whitby (1975) for the cylindrical DMA, and which will be summarized here for the parallel-plate geometry.

The equation of motion for the ion of mobility $k$ is

$\frac{d \boldsymbol{r}}{d t}=\boldsymbol{u}+k \boldsymbol{E}$,

where $\boldsymbol{r}$ is the ion position vector, $\boldsymbol{u}$ the gas flow velocity, and $\boldsymbol{E}$ the applied electric field. The main gas stream flows in the $x$ direction, and the electric field is directed along the $y$-coordinate, normal to the electrodes. Hence, (1) reduces to

$\frac{d x}{d t}=u_{x} ; \quad \frac{d y}{d t}=k E_{y}$.

Next, the stream function $\psi$ is defined by means of the relations

$u_{x}=-\frac{1}{\Delta_{z}} \frac{\partial \psi}{\partial y} ; \quad u_{y}=-\frac{1}{\Delta_{z}} \frac{\partial \psi}{\partial x}$,

where $\Delta_{z}$ is the DMA width. The latter is assumed to be sufficiently large so that the flow velocity $u_{x}$ depends only on $y$. In writing (3) it has been further assumed that the flow is incompressible in the classification zone, i.e. $\nabla \boldsymbol{u}=0$. Hence,

$\psi=-\Delta_{z} \int u_{x} d y$.

By analogy to $\psi$, we introduce the function

$\phi=\Delta_{z} \int E_{y} d x$,

which may be called the electric flux function.

Consider now the expression $T=\psi+k \phi$, and form its substantial derivative following the ion trajectory:

$\frac{D T}{D t}=\frac{\partial T}{\partial t}+\frac{d x}{d t} \frac{\partial T}{\partial x}+\frac{d y}{d t} \frac{\partial T}{\partial y}$.

For steady-state DMA operation, $\partial T / \partial t=0$. The last two terms in (6) cancel each other because of (2), (4) and (5). Therefore,

$T=\psi+k \phi=$ const.

along the ion trajectory.

In the classified stream withdrawn through the outlet slit there will appear ions with mobility distributed within two extreme values, which will be denoted as $k_{\min }$ and $k_{\max }$. These can be determined considering the boundary streamlines shown schematically in Fig. 2.

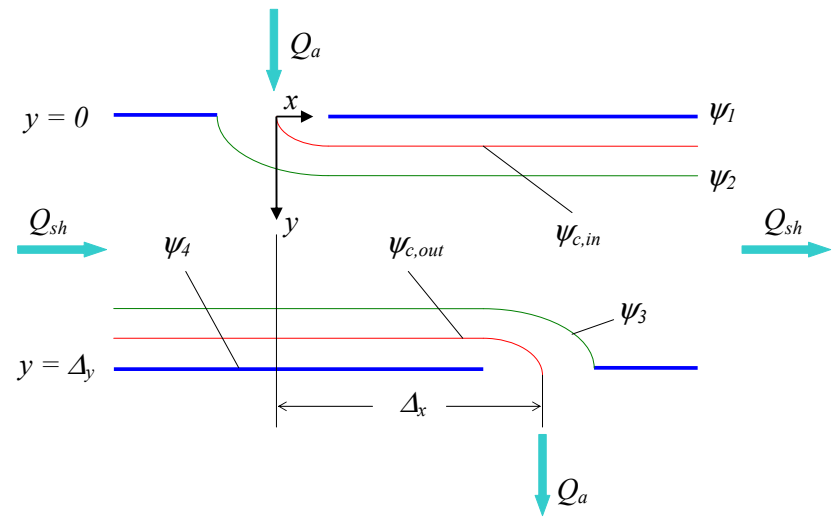

Fig. 2. Schematic representation of the streamlines involved in the determination of the DMA transfer function.

In the normal operation of the DMA, the flow rate of the incoming aerosol matches the flow rate of the classified stream, $Q_{a}$ and, likewise, the flow rates of the sheath gas entering and exiting the classification zone are equal to each other, and will be denoted as $Q_{s h}$. According to (4), the flow bounded by any two streamlines $\psi_{i}$ and $\psi_{k}$ is given by the difference $\psi_{i}-\psi_{k}$. Therefore, we can write

$Q_{a}=\psi_{1}-\psi_{2}=\psi_{3}-\psi_{4}$,

$Q_{s h}=\psi_{2}-\psi_{4}=\psi_{1}-\psi_{3}$.

The ion with minimum mobility which appears in the classified stream is that which enters the classification zone with streamline $\psi_{2}$ and is withdrawn along streamline $\psi_{3}$. For this ion, (7) becomes

$\psi_{2}+k_{\min } \phi_{\text {in }}=\psi_{3}+k_{\min } \phi_{\text {out }}$,

from where

$k_{\text {min }}=\frac{\psi_{2}-\psi_{3}}{\phi_{\text {out }}-\phi_{\text {in }}}$.

Neglecting edge effects in the electric field, we can write

$\phi_{\text {out }}-\phi_{\text {in }}=\frac{\Delta_{x} \Delta_{z}}{\Delta_{y}} V$,

where $V$ is the voltage applied between the electrodes, $\Delta_{y}$ is the separation distance between the electrodes, and $\Delta_{x}$ is the distance between the inlet and outlet slits measured along the $x$-direction. Substituting (11) into (10), and taking (8) and (9) into account, we arrive at

$k_{\min }=\frac{\Delta_{y}}{\Delta_{x} \Delta_{z}} \frac{Q_{s h}-Q_{a}}{V}$.

In an analogous manner, one can determine the maximum mobility of the classified ions. These enter the DMA with 
streamline $\psi_{1}$ and leave the classification zone along streamline $\psi_{4}$. After a few elementary algebraic manipulations, it is found

$k_{\max }=\frac{\Delta_{y}}{\Delta_{x} \Delta_{z}} \frac{Q_{s h}+Q_{a}}{V}$.

Finally, the mean mobility of the ions in the classification stream can be determined either repeating the above analysis for streamlines $\psi_{c \text {,in }}$ and $\psi_{c \text {, out }}$, or simply by taking the arithmetic mean of expressions (12) and (13):

$k_{c}=\frac{\Delta_{y}}{\Delta_{x} \Delta_{z}} \frac{Q_{s h}}{V}$.

The instrument resolution can be expressed as the dimensionless full width at half height (FWHH) of the transfer function:

$\mathrm{FWHH}_{\mathrm{id}}=\frac{\Delta k}{2 k_{c}}=\frac{k_{\max }-k_{\min }}{2 k_{c}}=\frac{Q_{a}}{Q_{s h}}$.

Thus, in ideal conditions, the resolution is given by the aerosol-to-sheath flow rate ratio.

At a given applied voltage, all the possible trajectories of an ion of mobility $k$ are bounded by two limiting trajectories, which will be called $T_{A}$ and $T_{B}$. From (7) we have

$\psi_{A, \text { in }}+k \phi_{\text {in }}=\psi_{A, \text { out }}+k \phi_{\text {out }}$,
$\psi_{B, \text { in }}+k \phi_{\text {in }}=\psi_{B, \text { out }}+k \phi_{\text {out }}$.

Consider again the schematic streamlines drawn in Fig. 2. The boundary trajectories for the ion of mobility $k$ start at the starting points of streamlines $\psi_{1}$ and $\psi_{2}$, say

$\psi_{A, \text { in }}=\psi_{2}, \quad \psi_{B, \text { in }}=\psi_{1}$.

With the help of (11), and (15)-(18), one finds the "outlet" boundary streamlines for this ion of mobility $k$ :

$\psi_{A, \text { out }}=\psi_{2}-\frac{\Delta_{x} \Delta_{z}}{\Delta_{y}} k V$

$\psi_{B, \text { out }}=\psi_{1}-\frac{\Delta_{x} \Delta_{z}}{\Delta_{y}} k V$

The transfer function can then be evaluated as the fraction of the flow $\psi_{B \text {,out }}-\psi_{A \text {,out }}$ which is common with (or "intersects") the classified flow $\psi_{3}-\psi_{4}$ :

$\Omega=\left\{\begin{array}{c}\frac{\min \left(\psi_{3}, \psi_{B, \text { out }}\right)-\max \left(\psi_{4}, \psi_{A, \text { out }}\right)}{\psi_{B, \text { out }}-\psi_{A, \text { out }}} \\ \text { if } \psi_{A, \text { out }} \psi_{3} \text { and } \psi_{B, \text { out }} \geq \psi_{4} . \\ 0 \text { elsewhere }\end{array}\right.$

The procedure to express (21) in terms of flow rates and mobilities is purely algebraic and straightforward, but tedious. The final result is

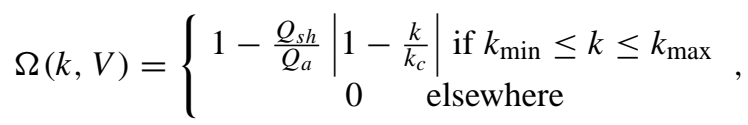

where $k_{\min }, k_{\max }$ and $k_{c}$ are given by expressions (12)-(14).

The transfer function (22) describes the ideal operation of the DMA. Its shape is a triangle of unit height, symmetric about the central mobility $k_{c}$, and with a base width equal to $k_{\max }-k_{\min }$. It is interesting to note that the transfer function (22) coincides exactly with that found for concentric cylindrical DMAs (Knutson and Whitby, 1975).

\subsection{Diffusion broadening of the transfer function}

In real conditions, the transfer function shape is closer to a Gaussian, with height less than unity and base width larger than $k_{\max }-k_{\min }$, because the strong Brownian motion of the ions prevents them from following the deterministic trajectories given by the simple Eq. (7). The distorting effect of Brownian motion on the shape of the transfer function has been studied by a number of authors (Tammet, 1970; Kousaka et al., 1985; Stolzenburg, 1988; Rosell-Llompart et al., 1996; Hagwood et al., 1999; Salm, 2000).

The quantitative effect of Brownian diffusion on the instrument resolution can be estimated in a particularly simple manner. In the absence of diffusion, an ion follows the trajectory given by the equation of motion (2) and arrives at the collector electrode at a certain position $x^{\prime}$ downstream. When diffusion is taken into account, the arrival position of the ion can be approximated as a Gaussian distribution of mean $x^{\prime}$ and standard deviation

$\sigma_{\text {diff }}=\frac{\delta}{\Delta_{x} \cos \alpha}$,

where $\delta=\sqrt{2 D t}$ is the root mean square displacement about the deterministic trajectory ( $D=$ ion diffusion coefficient; $t=$ mean ion residence time in the classification zone), $\alpha$ is the angle formed between this trajectory and the $y$-axis, and the effective length of the classification zone, $\Delta_{x}$, has been introduced simply to express $\sigma_{\mathrm{diff}}$ in dimensionless form. Expressing $t$ in terms of the mean axial velocity $\overline{u_{x}}\left(t=\Delta_{x} / \overline{u_{x}}\right)$, diffusional variance can be written as

$\sigma_{\text {diff }}^{2}=\frac{2 D}{\overline{u_{x}} \Delta_{x}} \frac{\Delta_{x}^{2}+\Delta_{y}^{2}}{\Delta_{y}^{2}}$.

On the other hand, using the Einstein's relation $k=p e D / k_{B} T$ ( $p e=$ total charge on the ion; $k_{B}=$ Boltzmann's constant; $T=$ absolute temperature), and expressing $\overline{u_{x}}$ in terms of the flow rates, it is straightforward to find, with the help of (14), that the relative diffusional variance can be also expressed as

$\sigma_{\text {diff }}^{2}=4 \frac{k_{B} T}{p e V} \frac{Q_{s h}}{Q_{s h}+Q_{a}} \frac{\Delta_{x}^{2}+\Delta_{y}^{2}}{2 \Delta_{x}^{2}}$

since in the IONER X1 the conditions $\Delta_{x}=\Delta_{y}$ and $Q_{a} \ll Q_{s h}$ are satisfied, the expression above reduces to

$\sigma_{\text {diff }}^{2}=4 \frac{k_{B} T}{p e V}$. 


\subsection{Combined effect of flow rate ratio and diffusion on the resolution}

Since variances are additive, it is possible to estimate the DMA resolution accounting for the combined effect of flow rate ratio and diffusion using the total relative variance

$\sigma^{2}=\sigma_{i d}^{2}+\sigma_{\text {diff }}^{2}$,

with $\sigma_{\text {diff }}^{2}$ given by (26), and

$\sigma_{i d}^{2}=\frac{\left\langle k^{2}\right\rangle_{i d}-\langle k\rangle_{i d}^{2}}{k_{c}^{2}}=\frac{1}{6}\left(\frac{\Delta_{y}}{\Delta_{x} \Delta_{z}} \frac{Q_{a}}{k_{c} V}\right)^{2}$,

where the moments of the classified ions mobility distribution have been calculated as

$$
\left\langle k^{m}\right\rangle_{i d}=\frac{\int_{k_{\min }}^{k_{\max }} k^{m} \Omega d k}{\int_{k_{\min }}^{k_{\max }} \Omega d k},
$$

and $\Omega$ is the ideal transfer function given by (22).

Assuming that the combined transfer function is a Gaussian, the full width at half height (FWHH) is related to the standard deviation $\sigma$ by the formula $\mathrm{FWHH}=(8 \ln 2)^{1 / 2} \sigma$, and one arrives at

$\mathrm{FWHH}=\left\{8 \ln 2\left[\frac{1}{6}\left(\frac{\Delta_{y}}{\Delta_{x} \Delta_{z}} \frac{Q_{a}}{k_{c} V}\right)^{2}+4 \frac{k_{B} T}{p e V}\right]\right\}^{1 / 2}$.

\subsection{Non-ideal behaviour}

Besides Brownian motion, other nonidealities may affect the DMA resolution. The theory on the ideal DMA assumes that the flow and the electric fields are fully uniform, unidirectional and perpendicular to each other along the entire length of the classification region. In this case, ion trajectories are properly described by the 2-D model presented in Sect. 2.1. However, the experience gained in cylindrical DMAs reveals that deviations from the ideal flow and electric fields in the classification zone can degrade severely the DMA performance. In particular, a lot of work has been dedicated to optimise the means (filters, meshes) to attain a laminar, unidirectional and uniform sheath flow as well as the design (inclination, width) of the ion inlet slit, which are major potential sources of loss of DMA resolution (Chen and Pui, 1997; Eichler et al., 1998; Chen et al., 1999). Nonidealities in planar DMAs have not been studied as extensively as for cylindrical DMAs. In this regard, a disadvantage of the DMAs of rectangular plates with respect to radial DMAs is the existence of lateral edges in the classification zone and in the ion inlet and outlet slits, which induce undesirable nonuniformities and 3-D features in the flow and electric fields.

Numerical and experimental studies have provided some insight into the edge effects in the IONER X1, although their impact on the performance of the instrument has not been fully assessed to date. On one side, the flow in the IONER $\mathrm{X} 1$ was simulated numerically by using a commercial CFD programme (FLUENT). The simulations show that, at the high Reynolds numbers of the flow in the classification zone $\left(R e>2 \times 10^{4}\right)$, the flow profile is quite flat expect in a thin region $(<2 \mathrm{~mm})$ over the surface of the channel side walls. In addition, the distribution of the ion sample flow over the length of the entrance slit was investigated experimentally. A $\mathrm{pH}$ indicator paper was fixed onto the electrode surface, immediately downstream of the inlet slit. A stream of nitrogen was passed through a bubbler containing a liquid sample of either clorhidric acid or ammonium and then introduced into the IONER X1 through the sample flow injector. In the classification zone the sample flow travels confined to a narrow region close to the electrode surface, so the vapour of clorhidric acid $(\mathrm{HCl})$ or ammonia $\left(\mathrm{NH}_{3}\right)$ diffuses to the surface of the indicator paper changing its colour accordingly. The spots on the paper provide a qualitative indication of the distribution of the sample flow over the slit. Experiments were performed at sheath flow rates in the range of 200 to $7001 \mathrm{~min}^{-1}$, sample flow rates between 2 and $61 \mathrm{~min}^{-1}$ and slit widths of 50, 100 and $150 \mu \mathrm{m}$. In all the cases the spots exhibited a rather uniform colour over the entire length of the ion inlet slit, except in the close vicinity of the edges.

It is noticed that the width of the electrodes of the IONER $\mathrm{X} 1$ is $10 \mathrm{~mm}$, while the length of the ion inlet and outlet slits carved on their surface is $6 \mathrm{~mm}$. The lateral walls of the rectangular channel and the edges of the slits are then separated by a distance of $2 \mathrm{~mm}$. This is a way to minimize the effect of the non-uniform three-dimensional flow and electric fields that develop near the corners between the side walls (Ertalyte insulating material, PTE) and the top and bottom walls (aluminium) of the flow channel on the trajectories of the ions. Finally, ion losses by diffusion (either lateral or in the direction of migration) to the walls play no role in the IONER X1. As the flow velocity is very high $\left(>100 \mathrm{~m} \mathrm{~s}^{-1}\right)$, the residence time of the ions in the classification region is less than $30 \mu \mathrm{s}$, whereas the time it takes the ions to reach the walls is much longer, in the order of $1 \mathrm{~s}$.

\section{Performance evaluation}

First, a series of measurements were carried out to check the validity of the isentropic flow assumption commented above. Negative air ions generated by passing filtered and dried room air through a corona discharge ionizer (RAMEM IONER CC08010) were fed into the DMA at a constant flow rate of $31 \mathrm{~min}^{-1}$. The flow rate of sheath air was varied between about 200 and $7001 \mathrm{~min}^{-1}$. Some representative spectra are shown in Fig. 3. The sheath flow rates that appear in the legend are those calculated for the classification zone from the flow measurements of the Venturi-type flowmeter upstream and assuming isentropic flow conditions. The 
Table 1. Calculated reduced mobility for the high-mobility peak of Fig. 3. $k_{c}$ is the mobility as calculated with (14); $k_{0}$ is the reduced mobility, calculated with (31) using the calculated values of pressure and temperature at the classification zone, shown in the second and third columns.

\begin{tabular}{ccccc}
\hline $\begin{array}{c}Q_{s h} \\
\left(1 \mathrm{~min}^{-1}\right)\end{array}$ & $\begin{array}{c}P \\
(\mathrm{hPa})\end{array}$ & $\begin{array}{c}T \\
\left({ }^{\circ} \mathrm{C}\right)\end{array}$ & $\begin{array}{c}k_{c} \\
\left(\mathrm{~cm}^{2} \mathrm{~V}^{-1} \mathrm{~s}^{-1}\right)\end{array}$ & $\begin{array}{c}k_{0} \\
\left(\mathrm{~cm}^{2} \mathrm{~V}^{-1} \mathrm{~s}^{-1}\right)\end{array}$ \\
\hline 215 & 935 & 22.7 & 2.330 & 1.985 \\
292 & 909 & 19.1 & 2.366 & 1.984 \\
370 & 882 & 16.9 & 2.398 & 1.966 \\
447 & 854 & 14.4 & 2.466 & 1.974 \\
515 & 826 & 12.1 & 2.603 & 2.032 \\
607 & 768 & 8.0 & 2.686 & 1.978 \\
671 & 728 & 6.0 & 2.843 & 1.999 \\
\hline
\end{tabular}

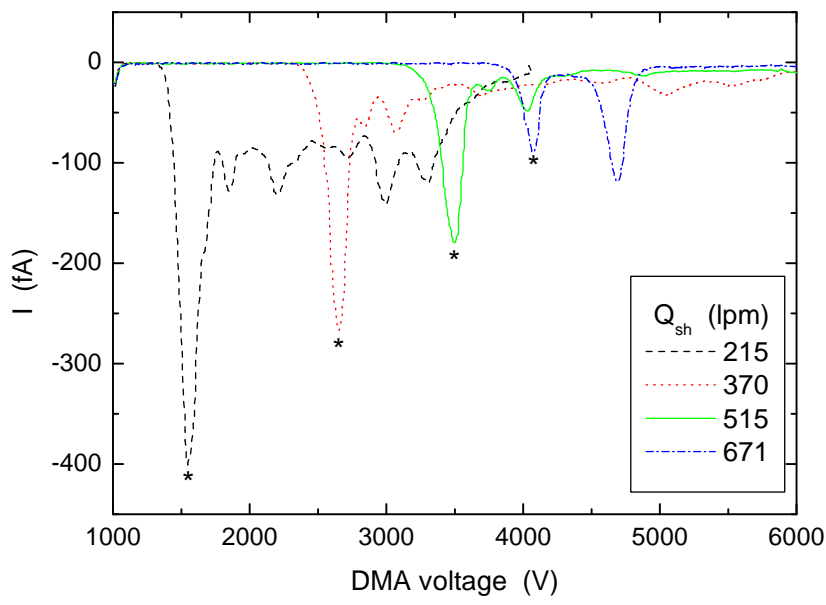

Fig. 3. Mobility spectra of negative air ions generated by corona discharge.

spectra are presented as the total ionic current measured by the electrometer placed behind the outlet slit versus the voltage difference applied to the parallel-plate electrodes. Table 1 shows the relevant data for the high-mobility peak, the one marked with an asterisk in Fig. 3. The mobility, calculated with (14), was transformed into reduced mobility by using Langevin's rule

$k_{0}=k_{c}\left(\frac{P}{760}\right)\left(\frac{273}{273+T}\right)$,

where $P$ (in $\mathrm{mmHg}$ ) and $T$ (in $\mathrm{K}$ ) are, respectively, the calculated pressure and temperature at the classification zone.

As the results in Table 1 show, the reduced mobilities, calculated from spectra obtained at quite different sheath flow rates, agree very well with each other. This example illustrates the degree of accuracy attainable with this spectrometer.

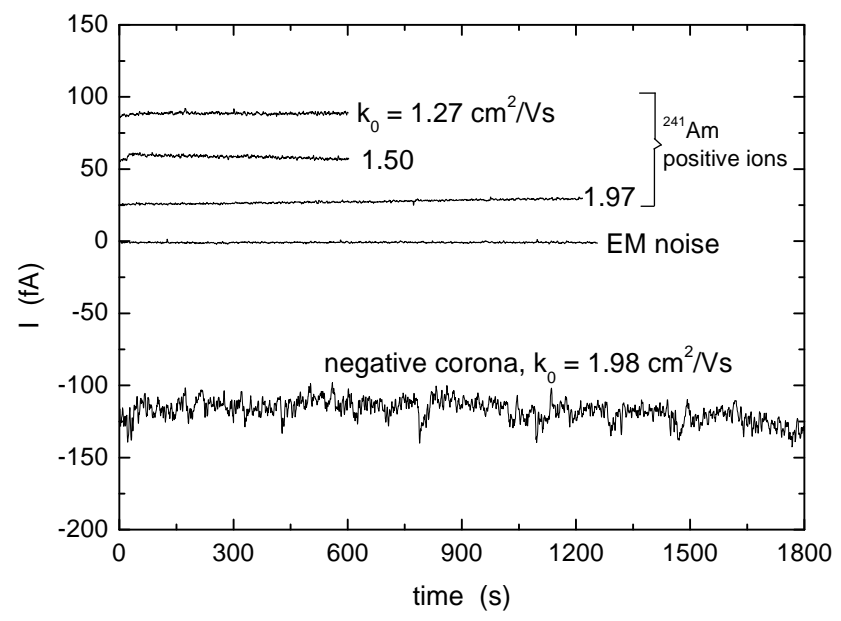

Fig. 4. Stability of DMA operating conditions and ion current measurement.

The repeatability of the measurements with the IONER X1 ion mobility analyzer has also proven to be excellent. The operating conditions (sheath and aerosol flow rates, voltage between the parallel-plate electrodes) as well as the electrometer response are very stable, as the data plotted in Fig. 4 shows. The results shown in this plot were obtained by recording the output current for periods of 10,20 and $30 \mathrm{~min}$, keeping fixed the DMA voltage and flow rates. Filtered and dried room air was passed through a small circular tube containing two thin disks of ${ }^{241} \mathrm{Am}$, each one with a nominal activity of $0.9 \mu \mathrm{Ci}$, stuck to the inner wall in diametrically opposite positions. The concentrations of positive ions generated by ${ }^{241} \mathrm{Am}$ were quite stable over long periods of time. The electrometer noise was of the order of $0.1 \mathrm{fA}$. In the case of negative corona air ions, those whose spectra was shown above in Fig. 3, the stability was not as good as for ions generated in the radioactive ionizer, but this is related to the corona ionizer itself rather than to the IONER X1 performance capability.

Experiments were conducted to assess the resolution of IONER X1 too. A solution of tetraheptylammonium bromide (THABr) in ethanol $\left(10^{3} \mathrm{ppm} w / \mathrm{v}\right)$ was electrosprayed (Fenn et al., 1989), resulting in two ions of tetraheptylammonium (THA) of well-defined electrical mobilities $\left(20^{\circ} \mathrm{C}, 1 \mathrm{~atm}\right)$ : $0.96 \mathrm{~cm}^{2} \mathrm{~V}^{-1} \mathrm{~s}^{-1}$ (monomer) and $0.67 \mathrm{~cm}^{2} \mathrm{~V}^{-1} \mathrm{~s}^{-1}$ (dimer), both carrying a single positive charge (Gamero-Castaño and Fernández de la Mora, 2000). A stream of nitrogen was used to help nebulize the liquid and evaporate the neutral solvent in the droplets. The flow of nitrogen carrying the THA ions was then injected to the IONER X1.

The ion flow rate $Q_{a}$ was maintained constant at $41 \mathrm{~min}^{-1}$, while the sheath flow rate $Q_{s h}$ was varied between 200 and $5401 \mathrm{~min}^{-1}$; the corresponding classification voltage $V$ ranged from 3.5 to $7.8 \mathrm{kV}$. The ion mobility peaks could be well fitted to Gaussians. The relative full width at half height 


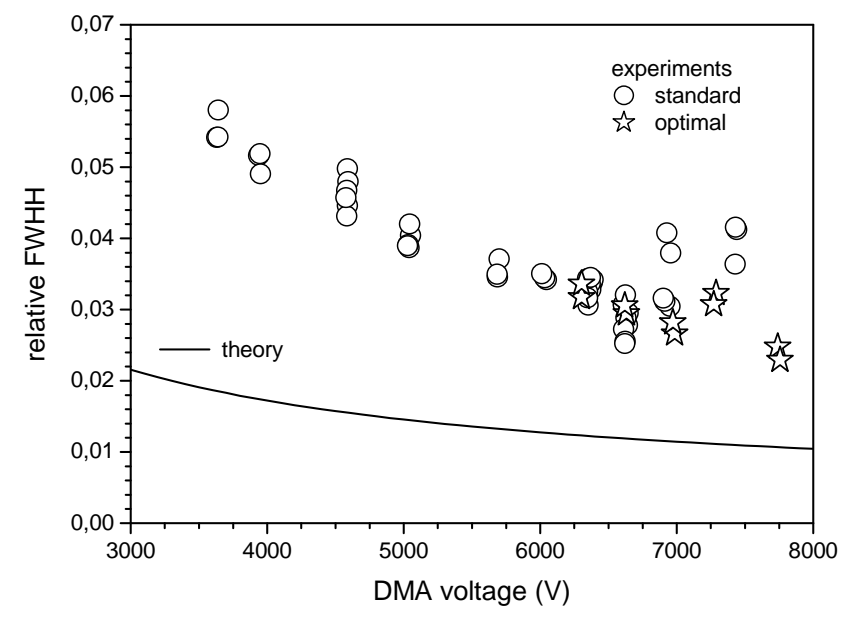

Fig. 5. Resolving power of IONER X1. Symbols: experimental results; line: curve calculated with (30).

(FWHH) of the mobility peaks corresponding to monomer THA $\left(k=0.96 \mathrm{~cm}^{2} \mathrm{~V}^{-1} \mathrm{~s}^{-1}\right)$ is plotted as a function of the classification voltage in Fig. 5; the theoretical values predicted by (30) are also shown for comparison. Several series of experiments were carried out in which the relative positions of the laminarizing screens were changed with respect to a standard configuration. The best resolution value of 0.025 was obtained at the maximum classification voltage $(V=7.8 \mathrm{kV})$, while the theory predicts a value of 0.011 .

The reasons for the deviations between experimental and theoretical values of the relative FWHH observed in Fig. 5 are presently not understood. They are attributed to nonideal behaviour of the flow and the electric fields in the classification zone of the IONER X1, potentially induced by the lateral walls, the edges of the ion slits, nonoptimal design of the ion sample injector and/or inlet slit, etc. Nonidealities in the IONER X1 have not been addressed in this work, and they will be the subject of a future work.

Relative FWHH values as low as 0.01 has been attained for THA ions with high-resolution cylindrical DMAs (MartínezLozano et al., 2005, 2006) in experiments similar to the ones previously described. Nonetheless, the IONER X1 and the cylindrical DMAs operated at different sheath and ion flow rates, thereby different flow rate ratios $Q_{s h} / Q_{a}$ and classification voltages $V$, which renders difficult the comparison of the resolving power of cylindrical and planar DMAs. In this regard, the lowest value of the relative FWHH for monomer THA found with the IONER X1 is limited by the maximum voltage attainable $(\sim 8 \mathrm{kV})$, above which electric spark occurred in the instrument during the experiments.

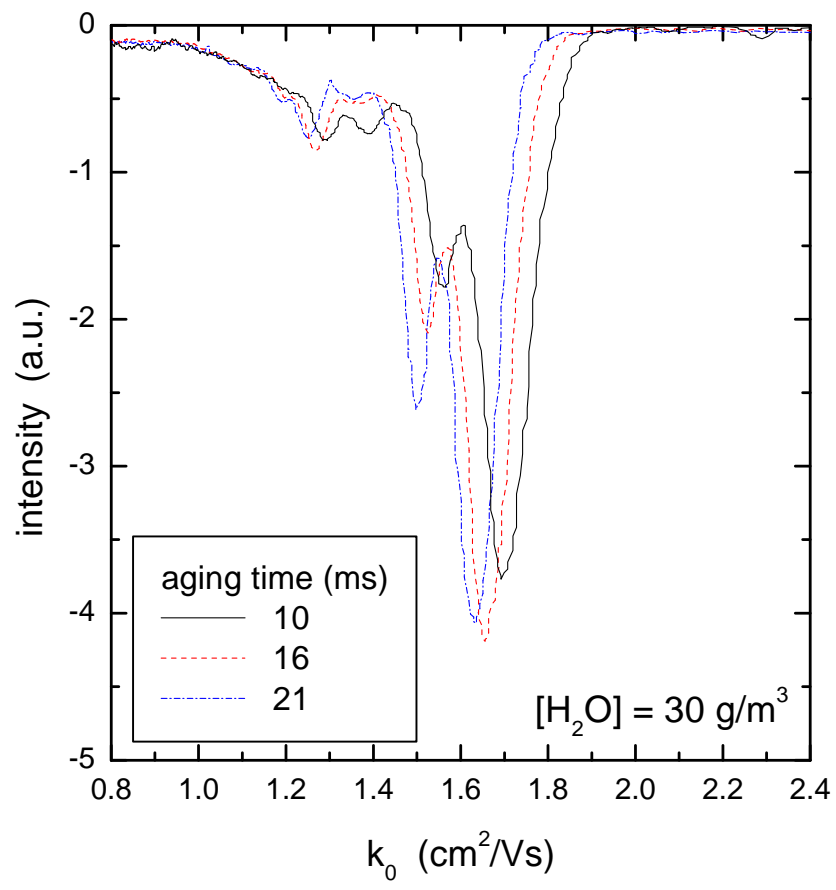

Fig. 6. Effect of aging time on the mobility distribution of negative air ions at constant humidity.

\section{Applications of the high-resolution differential mobil- ity analyzer}

In the final part of this article, we will present the results of some experimental measurements carried out with the aim to illustrate the potential capabilities of this novel instrument. The selected example deals with the effects of air humidity and aging time on the mobility spectra of air ions generated by a radioactive source.

Humid air with known water content was prepared by mixing, in variable proportions, a stream of synthetic air (water content below $3 \mathrm{ppm}$ according to the manufacturer, Air Liquide Alphagaz) with a second stream of synthetic air previously passed through a humidifier operated under controlled conditions of pressure and temperature. The resulting mixture was made to flow through the ${ }^{241} \mathrm{Am}$ ionizer and the DMA.

The volume between the outlet of the ionizer and the inlet slit of the DMA was $1.04 \mathrm{~cm}^{3}$. The aging time of ions was determined as their mean residence time in this volume. Aging time was varied by modifying the total flow rate of the mixture of dry and humid air passing through the ionizer into the DMA. Experiments were carried out with water content ranging between 0 (actually $<3 \mathrm{ppm}$ ) and $40 \mathrm{~g} \mathrm{~m}^{-3}$, and mean ion aging time between 10 and $25 \mathrm{~ms}$.

Figures 6 and 7 show the effect of aging time on the mobility distribution of negative and positive air ions at fixed water content. Mobilities are expressed as reduced mobilities at $0^{\circ} \mathrm{C}$ and $1 \mathrm{~atm}$, using (31). The spectra were normalized so 


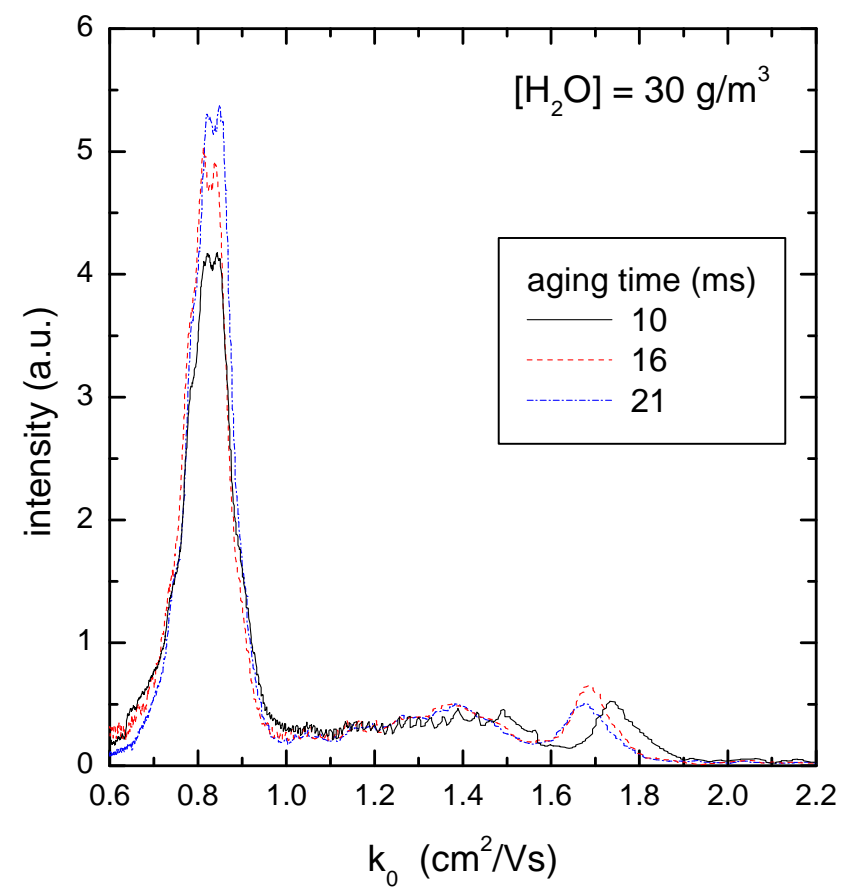

Fig. 7. Effect of aging time on the mobility distribution of positive air ions at constant humidity.

that the area under each curve is unity. In this manner, the relative area under each peak is directly related to the relative proportion of the corresponding chemical species in the mixture.

Ions grow with time due to, essentially, clustering processes. Specifically, when there is water molecules in the system, ions grow by progressive hydration, i.e. by attachment of additional water molecules. The change in mobility due to water clustering is more clearly noticeable in the case of high mobility ions: addition of one water molecule to an ion results in a relatively larger growth is the ion is small. Note that negative ions with mobility about $1.7 \mathrm{~cm}^{2} \mathrm{~V}^{-1} \mathrm{~s}^{-1}$ can grow into ions of mobility near $1.6 \mathrm{~cm}^{2} \mathrm{~V}^{-1} \mathrm{~s}^{-1}$ within $10 \mathrm{~ms}$. The rate at which the spectra peaks shifts toward lower mobility depends obviously on air humidity; mobility shifts were not so pronounced at lower water concentrations.

In the case of positive air ions (Fig. 7) a clear shift occurs only for the ions of higher mobility. Note, however, that the relative proportion of low mobility ions (those with mobility peaks about 0.80 and $0.85 \mathrm{~cm}^{2} \mathrm{~V}^{-1} \mathrm{~s}^{-1}$ increases with aging time, which is in agreement with the qualitative picture presented above. In general, at a given fixed air humidity, the mean mobility of the ion population decreases with aging time.

Figures 8 and 9 show some mobility spectra obtained at fixed aging time and variable air humidity. In comparison with the previous spectra showing the effect of ion aging time, it can be observed the drastic influence that water con-

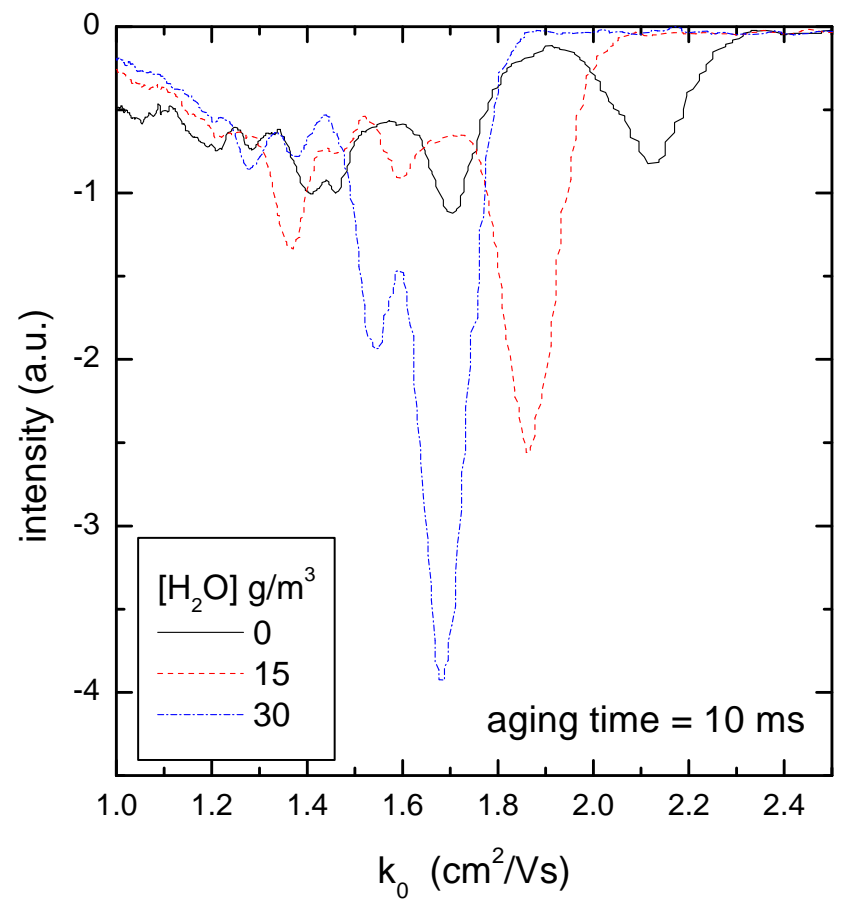

Fig. 8. Effect of air humidity on the mobility of negative air ions at fixed aging time.

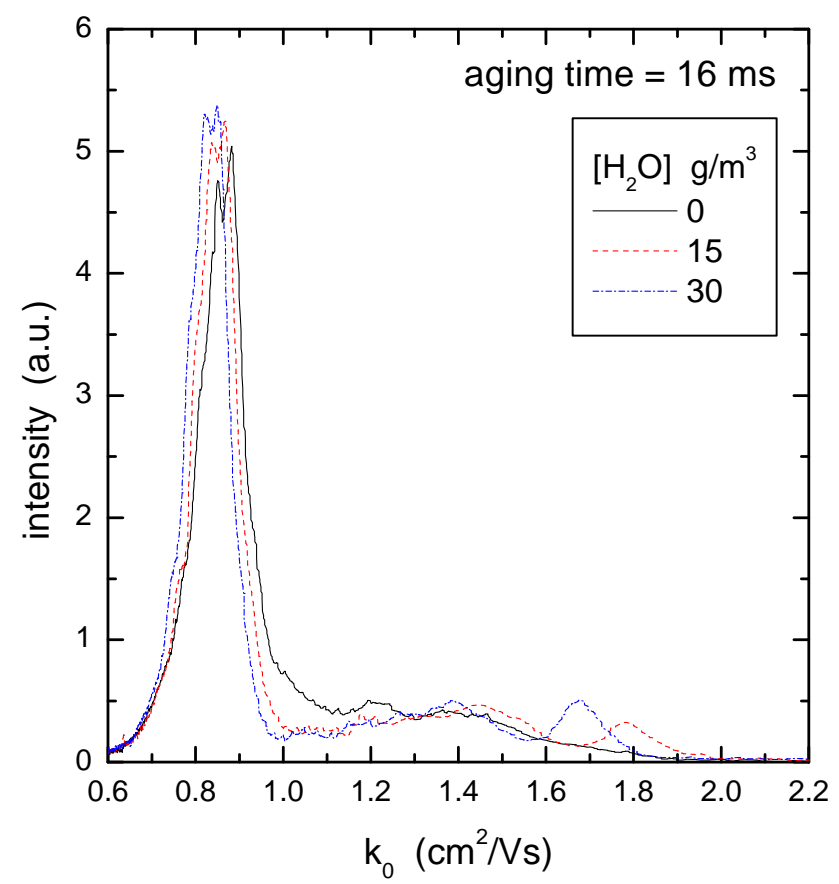

Fig. 9. Effect of air humidity on the mobility of positive air ions at fixed aging time.

centration has on the chemical nature of the ions. The presence of water even in trace amounts (as in the experiments in which no air was passed through the humidifier) suffices to 
promote water clustering (Reischl et al., 1996). Thus, for instance, the peak at $2.12 \mathrm{~cm}^{2} \mathrm{~V}^{-1} \mathrm{~s}^{-1}$ in the spectrum drawn with continuous line in Fig. 8, typical of an air ion containing a very few number of water molecules, completely disappears when air humidity rises to $15 \mathrm{~g} \mathrm{~m}^{-3}$, and an ion containing several more water molecules, with mobility of $1.87 \mathrm{~cm}^{2} \mathrm{~V}^{-1} \mathrm{~s}^{-1}$, appears in its place.

The changes in mobility are less remarkable in the case of positive ions for the reason explained before: large ions do not grow relatively much. Nevertheless, the growth of low mobility positive ions into large ions is also observable as air humidity increases.

Sheath gas temperature changes were below $0.5^{\circ} \mathrm{C}$ in all experiments above except for the spectra in Fig. 6 where the temperature increased $1.8^{\circ} \mathrm{C}$ along the measurements which corresponds to a decrease of less than $9 \%$ in relative humidity from the first spectrum to the last one.

\section{Conclusions}

A novel, high-resolution differential mobility analyzer, specially indicated for the measurement of mobility spectra in the range $0.5-3.0 \mathrm{~cm}^{2} \mathrm{~V}^{-1} \mathrm{~s}^{-1}$, has been developed, manufactured, and successfully tested. The novelty of the instrument resides in its parallel-plate geometry, which avoids the traditional electrode centering problem of conventional cylindrical DMAs. As with other DMAs, the apparatus described in this work permits, not only the measurement of mobility distributions, but the generation of a continuous stream of monomobile ions as well.

Edited by: S. Pandis

\section{References}

Alonso, M.: Reducing the diffusional spreading rate of a Brownian particle by an appropriate non-uniform external force field, J. Aerosol Sci., 33, 439-450, 2002.

Brunelli, N. A., Flagan, R. C., and Giapis, K. P.: Radial differential mobility analyzer for one nanometer particle classification, Aerosol Sci. Technol., 43, 53-39, 2009.

Buryakov, I. A.: Express analysis of explosives, chemical warfare agents and drugs with multicapillary column gas chromatography and ion mobility spectrometry, J. Chromatography B, Anal. Technol. Biomedical Life Sci., 800, 75-82, 2004.

Chen, Y. H., Hill, H. H., and Wittmer, D. P.: Thermal effects on electrospray ionization ion mobility spectrometry, Int. J. Mass Spectrom. Ion Proc., 154, 1-13, 1996.

Chen, D. R. and Pui, D. Y. H.: Numerical modeling of the performance of differential mobility analyzers for nanometer aerosol measurements, J. Aerosol Sci., 28, 985-1004, 1997.

Chen, D.R., Pui, D.Y.H., Humes, D., Fissan, H., Quant, F.R., and Sem, G.J.: Design and evaluation of a nanometer aerosol differential mobility analyzer (Nano-DMA), J. Aerosol Sci., 29, 497509, 1998.
Chen, D. R., Pui, D. Y. H., Mulholland, G. W., and Fernandez, M.: Design and testing of an aerosol/sheath inlet for high resolution measurements with a DMA, J. Aerosol Sci., 30, 983-999, 1999.

Clemmer, D. E. and Jarrold, M. F.: Ion mobility measurements and their applications to clusters and biomolecules, J. Mass Spectrom., 32, 577-592, 1997.

de Juan, L. and Fernández de la Mora, J.: High resolution size analysis of nanoparticles and ions: Running a Vienna DMA of near optimal length at Reynolds numbers up to 5000, J. Aerosol Sci., 29, 617-626, 1998.

Eichler, T., de Juan, L., and Fernández de la Mora, J.: Improvement of the resolution of TSI's 3071 DMA via redesigned sheath air and aerosol inlets, Aerosol Sci. Technol., 29, 39-49, 1998.

Erikson, H. A.: The change of mobility of the positive ions in air with age, Phys. Rev. 18, 100-101, 1921.

Fenn, J. B., Mann, M., Meng, C. K., Wong, S. F., and Whitehouse C. M.: Electrospray ionization for mass spectrometry of large biomolecules, Science, 246, 64-71, 1989.

Fissan, H., Pocher, A., Neumann, S., Boulaud, D., and Pourprix, M.: Analytical and empirical transfer functions of a simplified spectrometre de mobilite electrique circulaire (SMEC) for nano particles - a theoretical study, J. Aerosol Sci., 29, 289-293, 1998.

Flagan, R. C.: History of electrical aerosol measurements, Aerosol Sci. Technol., 28, 301-380, 1998.

Gamero-Castaño, M. and Fernández de la Mora, J.: Mechanisms of electrospray ionization of singly and multiply charged salt clusters, Anal. Chim. Acta, 406, 67-91, 2000.

Hagwood, C., Sivathanu, Y., and Mulholland, G.: The DMA transfer function with Brownian motion: A trajectory/Monte-Carlo approach, Aerosol Sci. Technol., 30, 40-61, 1999.

Hill Jr., H. H. and Martin, S. J.: Conventional analytical methods for chemical warfare agents, Pure Appl. Chem., 74, 2281-2291, 2002.

Karpas, Z., Cohen, M. J., Stimac, R. M., and Wernlund, R. F.: On the effects of structure and charge distribution on the mobility of ions, Int. J. Mass Spectrom. Ion Processes, 74, 153-159, 1986.

Karpas, Z., Stimac, R. M., and Rappoport, Z.: Differentiating between large isomers and derivation of structure information by ion mobility spectrometry/mass spectrometry techniques, Int. J. Mass Spectrom. Ion Proc., 83, 163-175, 1988.

Knutson, E. O. and Whitby, K. T.: Aerosol classification by electric mobility: Apparatus, theory, and applications, J. Aerosol Sci., 6, 443-451, 1975.

Kousaka, Y., Okuyama, K., and Mimura, T.: Effect of Brownian diffusion on electrical classification of ultrafine particles in differential mobility analyzers, J. Chem. Eng. Jpn., 19, 401-407, 1986.

Langevin, P.: Sur la mobilitè des ions dans les gaz, Conzptes rendus, 134, 646-649, 1902.

Langevin, P.: L'ionization des gaz, Ann. Chim. Phys. 28, 289-384, 1903.

Loscertales, I. G.: Drift differential mobility analyzer, J. Aerosol Sci., 29, 1117-1139, 1998.

Martínez-Lozano, P. and Fernández de la Mora, J.; Resolution improvements of a nano-DMA operating transonically, J. Aerosol Sci., 37, 500-512, 2005.

Martínez-Lozano, P., Labowsky, M., and Fernández de la Mora, J.: Experimental tests of a nano-DMA with no voltage change between aerosol inlet and outlet slits, J. Aerosol Sci., 37, 1629- 
1642, 2006.

Ramiro, E., and Rivero, A.: Wide range differential mobility analyzer (DMA) with very high resolution, International Patent WO 2007/020303 A1, 22 February 2007.

Reischl, G. P., Mäkelä, J. M., Karch, R., and Necid, J.; Bipolar charging of ultrafine particles in the size range below $10 \mathrm{~nm}, \mathrm{~J}$. Aerosol Sci., 27, 931-949, 1996.

Rohmann, H.: Methode für Messung der Grösse von Schwebeteilchen. Z. Phys., 17, 253-265, 1923.

Rosell-Llompart, J., Loscertales, I. G., Bingham, D., and Fernández de la Mora, J.: Sizing nanoparticles and ions with a short differential mobility analyzer, J. Aerosol Sci., 27, 695-719, 1996.

Rosser, S. and Fernández de la Mora, J.: Vienna-type DMA of high resolution and high flow rate, Aerosol Sci. Technol., 39, 11911200, 2005.

Salm, J.: Diffusion distortions in a differential mobility analyzer: the shape of apparent mobility spectrum, Aerosol Sci. Technol., 32, 602-612, 2000.

Song, D. K. and Dhaniyala, S.: Change in distributions of particle positions by Brownian diffusion in a non-uniform external field, J. Aerosol Sci., 38, 444-454, 2007.

Stolzenburg, M.: An ultrafine aerosol size distribution measuring system, Ph.D. Thesis, University of Minnesota, USA, 1988.

Swing, R. G., Atkinson, D. A., Eiceman, G. A., and Ewin, G. J.: A critical review of ion mobility spectrometry for the detection of explosives and explosive related compounds, Talanta, 54, 515$529,2001$.
Tammet, H. F.: The aspiration method for the determination of atmospheric-ion spectra, Keter Press, Jerusalem, Israel, 1970.

Tammet, H., Mirne, A., and Tamm, E.: Electrical aerosol spectrometer of Tartu University, Atmos. Res., 62, 315-324, 2002.

Vautz, W., Zimmerman, D., Hartmann, M., Baumbach, J. I., Nolte, J., and Jung, J.: Ion mobility spectrometry for food quality and safety, Food Additives and Contaminants, 23, 1064-1073, 2006.

Whitby, K. T. and Clark, W. E.: Electrical aerosol particle counting and size distribution measuring system for the 0.015 to $1 \mu$ size range, Tellus, 18, 573-586, 1966.

Winklmayr, W., Reischl, G. P., Lidner, A. O., and Berner, A.: A new electromobility spectrometer for the measurement of aerosols size distributions in the size range from 1 to $1000 \mathrm{~nm}$, J. Aerosol Sci., 22, 289-296, 1991.

Zeleny, J.: On the ratio of the velocities of the two ions produced in gases by Röntgen radiation; and on some related phenomena, Phil. Mag., 46, 120-154, 1898.

Zhang, S.-H., Akutsu, Y., Russell, L. M., Flagan, R. C., and Seinfeld, J. H.: Radial differential mobility analyzer, Aerosol Sci. Technol., 23, 357-372, 2005.

Zhang, S.-H. and Flagan, R. C.: Resolution of the radial differential mobility analyzer for ultrafine particles, J. Aerosol Sci., 27, 1179-1200, 1996.

Zhang, M. and Wexler, A. S.: Cross flow ion mobility spectrometry: Theory and initial prototype testing, Int. J. Mass Spectrom., 258, 13-20, 2006 